\title{
Coupled Feedforward and Feedback Control Strategy of Parallel Hybrid Filter for Harmonic Mitigation
}

\author{
J. Škramlík ${ }^{1}$, V. Valouch ${ }^{1}$ \\ ${ }^{1}$ Institute of Electrical Engineering, Dolejškova 5, 18202 Prague 8, Czech Republic \\ E-mail: skramlik@iee.cas.cz, valouch@iee.cas.cz
}

\begin{abstract}
The strategy features a small sensitivity against a disturbed supply voltage system and enables us to set grid phase currents in the phase with respective voltages. The expected properties of the PHF control system have been confirmed by experimental tests in the laboratory. The data show that the modified control strategy yields better results than those obtained by using control systems reported previously, especially in case of an unsymmetrical and non-sinusoidal voltage system and the demand of zero reactive power withdrawn from the grid.
\end{abstract}

\section{Key words}

Parallel hybrid filter, feedforward and feedback control strategy, unsymmetrical voltage system, reactive power compensation, DSP control system.

\section{Introduction}

Passive filters (PF) are intended preferably for the compensation of the lagging power factor of a load and/or selected harmonics of its current. Active power filters (APF) may substitute the passive ones in some cases or serve as an additional tool, together with PF, for improving the harmonic spectra of the load current and supply voltage. In such a case we call that configuration a hybrid filter (HF) [1], [2].

In [3] a $3.3 \mathrm{kV}$ transformerless $\mathrm{AC} / \mathrm{DC}$ power conversion system is presented. The system consists of a three-phase diode rectifier and a parallel hybrid filter (PHF). The parallel hybrid filter is formed by a passive LC filter and a small active power filter connected in series without any transformer. A control strategy has been proposed that combines a feedback and a feedforward loop. The feedback grid current control loop is aimed at mitigation of all diode rectifier current harmonics whereas the feedforward load current loop is tuned only on the dominant fifth harmonic to improve filtering characteristics of the PHF.

Nevertheless, the control system proposed in [3] cannot guarantee that the fundamental grid current will be in the phase with the fundamental supply voltage. The presence of the fifth harmonic voltage at the point of the PHF coupling has strong unsuitable influence on the proper function of the feedforward loop as well.
In the paper a modified control algorithm of the PHF is presented, which features a small sensitivity against a disturbed supply voltage system and enables us to set grid phase currents in the phase with respective voltages.

\section{Parallel hybrid filter}

The power circuit with the PHF is shown in Fig. 1. At the front side of the rectifier an inductance $L_{\mathrm{AC}}$ is inserted to make is possible to change the type of the load to the current harmonic source instead of the harmonic voltage source. The PHF consists of the series combination of the $\mathrm{PF}$ tuned at the seventh harmonic and the APF with a voltage DC side. The digital control unit, based on the dSPACE control system DS 1103, generates firing signals for the APF. The source and load currents are detected as well as the voltage at the point of common coupling (PCC) and the DC voltage $v_{\mathrm{dc}}$. The input signals are the reference voltage for this DC voltage and demanded reactive power withdrawn from the grid.

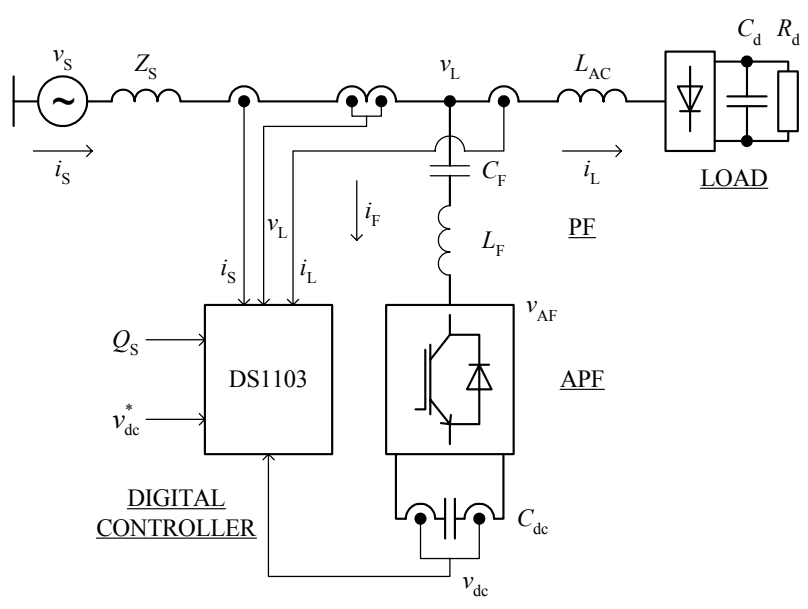

Fig. 1. Power circuit with PHF

\section{Feedback and feedforward control strategies}

Fig. 2 shows the proposed control scheme of the PHF. In the upper part of the figure the block diagram of the feedback control of the PHF is presented, while in the lower part the feedforward control algorithm is shown. 


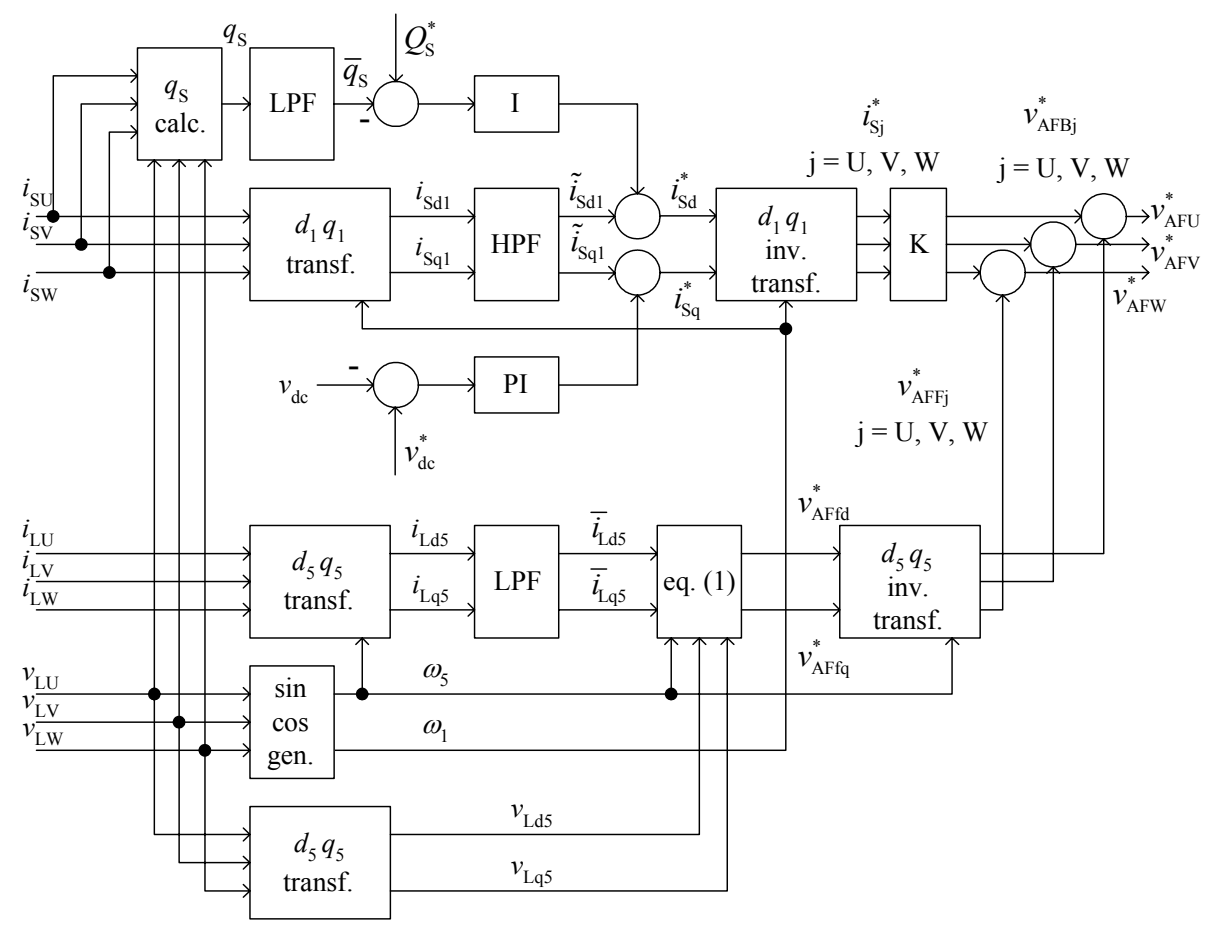

Fig. 2. Control scheme of the PHF

The scheme of the feedforward control is presented in the lower part of Fig. 2. Three load phase currents are transformed in their two orthogonal components in the reference frame rotating by the synchronous angular speed $\omega_{5}$ of the fifth harmonic. By using a low pass filter (LPF) of the first order with the cut-off frequency $f_{\mathrm{c}}=16$ $\mathrm{Hz}$ average $\mathrm{DC} d q_{5}$ components of the load current are obtained. These components should be delivered by the APF into the PCC instead of withdrawing them from the grid.

The reference voltage for the APF, which should be generated by the feedforward control loop, is then calculated as follows

$$
\boldsymbol{v}_{\mathrm{AFf}}^{*}=\boldsymbol{v}_{\mathrm{L} 5}-\left[R_{\mathrm{F}}+\mathrm{j}\left(\omega_{5} L_{\mathrm{F}}-\frac{1}{\omega_{5} C_{\mathrm{F}}}\right)\right] \overline{\boldsymbol{i}_{\mathrm{L} 5}}
$$

The reference $d q_{5}$ voltage components $\boldsymbol{v}_{\mathrm{AFfd}}^{*}, \boldsymbol{v}_{\mathrm{AFfq}}^{*}$ are then transformed back into the phase reference quantities. The final reference phase voltages for the APF are determined by summing the references coming from the feedback and feedforward loops.

\section{Experimental results}

No additional inductance $L_{\mathrm{AC}}$, converting the diode rectifier to the current harmonic source instead of the voltage harmonic source, was used [4].

The control system DS1103 is determined especially for rapid prototyping of digital controllers working with sophisticated algorithms. The core of the system is the RISC processor Motorola PowerPC 604e. For faster I/O communication the system is equipped with the additional processor Texas Instruments TMS320F240 DSP. The whole system is located on one standard PC/AT board.

Fig. 3 shows the captured phase supply voltages and currents when the control algorithm of the APF presented in Fig. 2 is in function. Fig. 4 presents phasor diagrams for the fundamental harmonics of the phase supply voltages and currents. It is evident that supply voltages as well as load currents represent highly unsymmetrical and non-sinusoidal systems, while supply phase currents involve a small amount of harmonics and are in phases with respective voltages. Due to the demand of zero reactive power withdrawn from the grid and due to the fact that the supply voltage system is unsymmetrical, the supply current system is not symmetrical too, neither in amplitudes nor in phases.

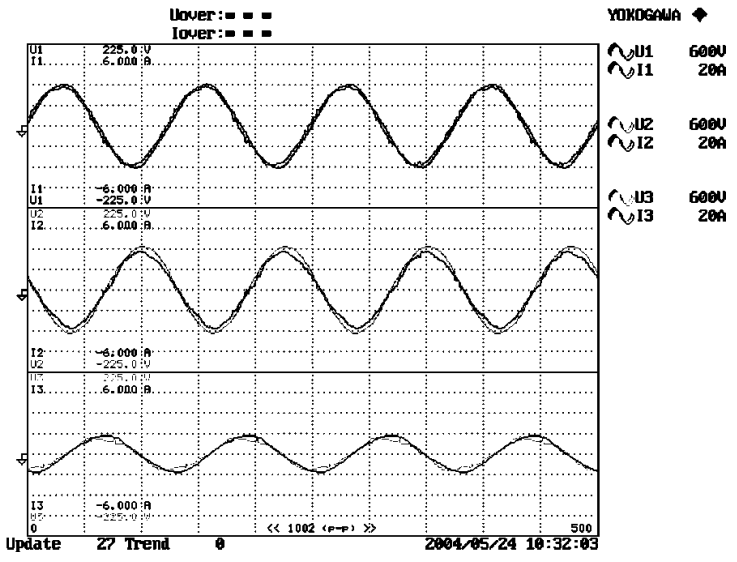

Fig. 3. Measured phase supply voltages and currents 
TABLE I. Harmonic spectra and the total harmonic distortion (THD) of the supply currents, expressed as the harmonic-to-fundamental current ratio (\%)

\begin{tabular}{|c|c|c|c|c|c|c|c|c|c|c|c|}
\hline PHF & Phase & $3^{\text {rd }}$ & $\overline{5}^{\text {th }}$ & $7^{7^{\text {th }}}$ & $\begin{array}{l}9^{\text {th }} \\
\end{array}$ & $11^{\text {th }}$ & $13^{\text {th }}$ & $15^{\text {th }}$ & $17^{e^{\text {th }}}$ & $19^{\text {th }}$ & THD $i_{\mathrm{s}}$ \\
\hline \multirow{3}{*}{ OFF } & $\overline{\mathrm{UU}}$ & 12.7 & 6.53 & 8.60 & 5.01 & 2.39 & 1.85 & 1.34 & 1.96 & 2.19 & 18.4 \\
\hline & $\mathrm{V}$ & 13.0 & 5.85 & 8.65 & 5.22 & 2.16 & 1.85 & 1.41 & 1.75 & 2.18 & 18.3 \\
\hline & W & 21.3 & 20.4 & 6.73 & 3.96 & 0.54 & 0.32 & 0.19 & 1.76 & 0.34 & 30.6 \\
\hline \multirow{3}{*}{$\mathrm{ON}$} & $\mathrm{U}$ & 1.17 & 0.34 & 1.51 & 0.51 & 0.61 & 0.72 & 0.20 & 0.49 & 0.44 & 4.1 \\
\hline & V & 2.31 & 0.27 & 1.83 & 0.45 & 0.39 & 0.84 & 0.56 & 0.54 & 0.40 & 5.0 \\
\hline & W & 4.21 & 0.22 & 0.71 & 0.23 & 0.61 & 0.18 & 0.85 & 0.27 & 0.43 & 5.0 \\
\hline
\end{tabular}

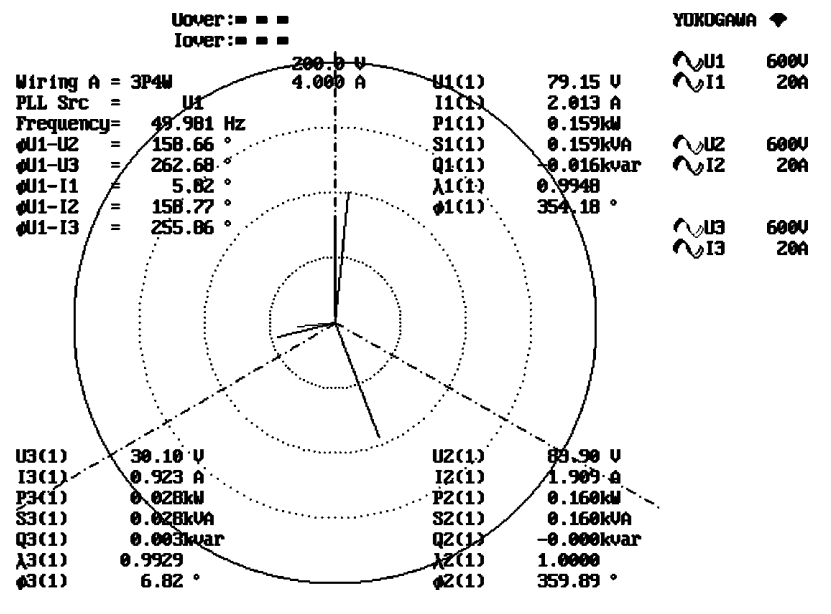

Fig. 4. Phasor diagrams for the fundamental harmonics of the phase supply voltages and currents

The harmonic spectra and the total harmonic distortion (THD) of the supply currents are summarized in Tab. I for the whole PHF being off and on.

\section{Conclusion}

In the paper the combined feedback and feedforward control strategy of the PHF is presented, which features a small sensitivity against a disturbed supply voltage system and enables us to set grid phase currents in the phase with respective voltages. The expected properties of the PHF control system have been confirmed by experimental tests in the laboratory.
The experimental data show that the modified control strategy yields better results than those obtained by using the original control system reported in [3], especially in case of an unsymmetrical and non-sinusoidal voltage system and the demand of zero reactive power withdrawn from the grid.

\section{Acknowledgement}

The financial supports of the Grant Agency of the Czech Republic (project No. 102/03/1363) and the Grant Agency of the Academy of the Sciences of the Czech Republic (project No. A2057301) are highly acknowledged.

\section{References}

[1] Akagi, H.: New Trends in Active Filters for Power Conditioning, IEEE Transactions on IA-32, 1996, No. 6, pp. 1312-1322.

[2] Peng, F. Z.: Harmonic Sources and Filtering Approaches, IEEE IA Magazine, July/August 2001, pp. 18-25.

[3] Srianthumrong, S., Akagi, H.: A Medium-Voltage Transformerless AC/DC Power Conversion System Consisting of a Diode Rectifier and a Shunt Hybrid Filter, IEEE Transactions on IA-39, 2003, No. 3, pp. 874-882.

[4] Peng, F. Z.: Application Issues of Active Power Filters, IEEE IA Magazine, September/October 1998, pp. 21-30. 